\title{
Undescended Testicular Seminoma Presenting as an Intra-Abdominal Mass - A Case Report
}

\author{
Shankey Garg ${ }^{1}$, Dhruv Narayan², Kritika Agrawal ${ }^{3}$, Harneet Narula ${ }^{4}$, Sai Krishna Reddy ${ }^{5}$ \\ 1, 2, 3, 4, 5 Department of Radio -Diagnosis, Maharishi Markandeshwar Institute of Medical Sciences and Research, \\ MMDU, Mullana, Haryana, India.
}

\section{INTRODUCTION}

Cryptorchidism is a common congenital malformation of the male genitourinary tract. The malignant potential of cryptorchidism in undescended testes is ten percent with the risk being highest in intra-abdominally located testes. In our case report, a 25-year-old man presented with a lower abdominal mass, which was nontender and of two months duration. Physical examination of the scrotum revealed an empty left scrotum and inguinal canal. Computed tomography scan, exploratory laparotomy and histopathological examination revealed an intrabdominal testicular seminoma (germ cell tumour). The purpose of our case report is to highlight the need for routine scrotal examination in addition to routine physical examination in all men who present with an abdominal mass to rule out the possibility of an intraabdominal testicular neoplasm.

Cryptorchidism is defined as the absence of one or both testes from the scrotal sac and is one of the most frequently encountered birth defects of the male genitalia. ${ }^{1}$ The prevalence amongst full-term neonates is three percent and $30 \%$ in preterm neonates. ${ }^{2}$ There is a higher potential for malignant transformation for cryptorchid testes than normally descended testes. They also predispose to ischemia and infertility later in adulthood. ${ }^{3}$ Seminoma (germ cell tumour) is the most common malignant transformation of an undescended testis. ${ }^{4}$ A 25 -year-old male complaining of a painless, slow-growing abdominal mass found to have intraabdominal testicular seminoma in an undescended testis and confirmed histopathologically, is presented in this case report. We wanted to highlight the importance of considering testicular germ cell tumours in the abdomen and the importance of performing a groin examination in a patient presenting with an abdominal lump with an empty scrotal sac.
Corresponding Author: Dr. Dhruv Narayan,

Maharishi Markandeshwar Institute of Medical Sciences and Research, MMDU, Mullana, Mullana University Road, Ambala-133207, Haryana, India. E-mail:dnarayan457@gmail.com

DOI: $10.14260 /$ jemds/2022/85

How to Cite This Article:

Garg $S$, Narayan D, Agrawal $K$, et al. Undescended testicular seminoma presenting as an intra-abdominal mass - a case report. J Evolution Med Dent Sci 2022;11(03):436-438, 10.14260/jemds/2022/85

Submission 10-01-2022,

Peer Review 05-02-2022,

Acceptance 11-02-2022,

Published 25-02-2022.

Copyright (C) 2022 Shankey Garg et al. This is an open access article distributed under Creative Commons Attribution License [Attribution 4.0 International (CC BY 4.0)] 


\section{Case Report}

A 25-year-old man presented with a history of a slowly growing mass in the abdomen for two months not associated with abdominal pain, nausea, vomiting or bowel/bladder abnormalities. He also had a history of chronic renal calculi, undescended left testis and opioid dependence.

The patient was clinically stable with a soft, non-tender and non-distended abdomen. Physical examination revealed an empty left scrotum with normal blood tests.

A contrast-enhanced CT scan (CECT) revealed a large heterogeneous attenuation mass measuring approximately $16.5 \times 10.2 \mathrm{~cm}$ in the abdomen in the umbilical and hypogastric region extending superiorly up to the left lumbar region (Figure 1). The mass showed moderate post-contrast enhancement with non-enhancing areas of central necrosis and also a few foci of calcification on the plain scan. An engorged vascular pedicle was seen on the inferolateral aspect of mass on the left side from which the feeding artery could be traced to the abdominal aorta, at the level of the renal artery and an engorged draining vein could be seen draining into the prominent left renal vein. Also, an engorged pampiniform plexus could be seen extending from mass into the deep inguinal ring and inguinal canal reaching until the superficial inguinal ring. The empty left scrotal sac was appreciated with no evidence of left testis within it. Based on these findings, an inference of intraabdominal tumour of undescended left testis was made.
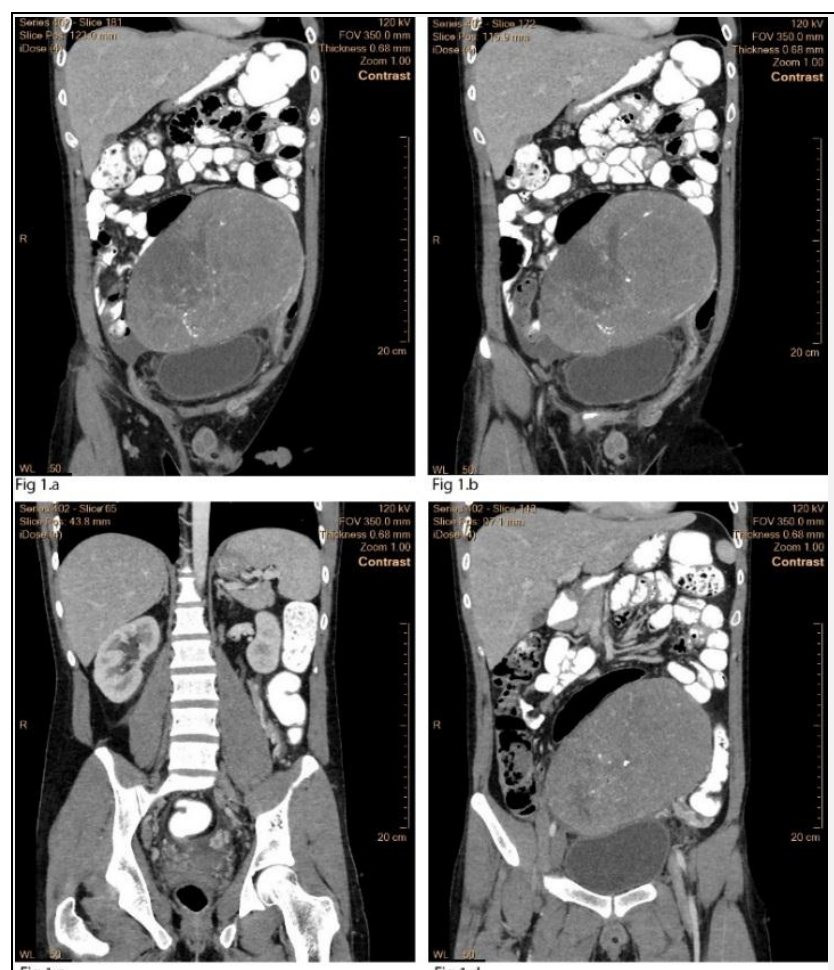

Figure 1. Contrast-Enhanced CT Images. 1. a: Coronal Image Showing Absent Spermatic Cord in the Left Inguinal Region. 1. b: Coronal Image Showing Engorged Vascular Pedicle Arising from the Inferolateral Aspect of Abdominal Mass. 1. c: Coronal Image Showing Vascular Pedicle till Abdominal Aorta and IVC. 1.d: Coronal Image Showing an Abdominal Mass in Umbilical and Hypogastric Region

A radiotherapy consultation was made wherein the patient received three cycles of neoadjuvant chemotherapy with a BEP regimen.
Finally, he underwent exploratory laparotomy and enbloc resection of the tumour. Intraoperative findings revealed a highly vascular mass, fixed to the left inguinal ligament and projecting into the retroperitoneum (Figure 2). Multiple nodal deposits were seen in the left paraaortic region. Mass effect was appreciated with superior displacement of bowel loops and indentation on bladder dome and rectosigmoid colon. Complete resection of the tumour was done within generous margins of normal tissue. The surgical specimen was sent for histopathological examination where a trucut biopsy was performed.

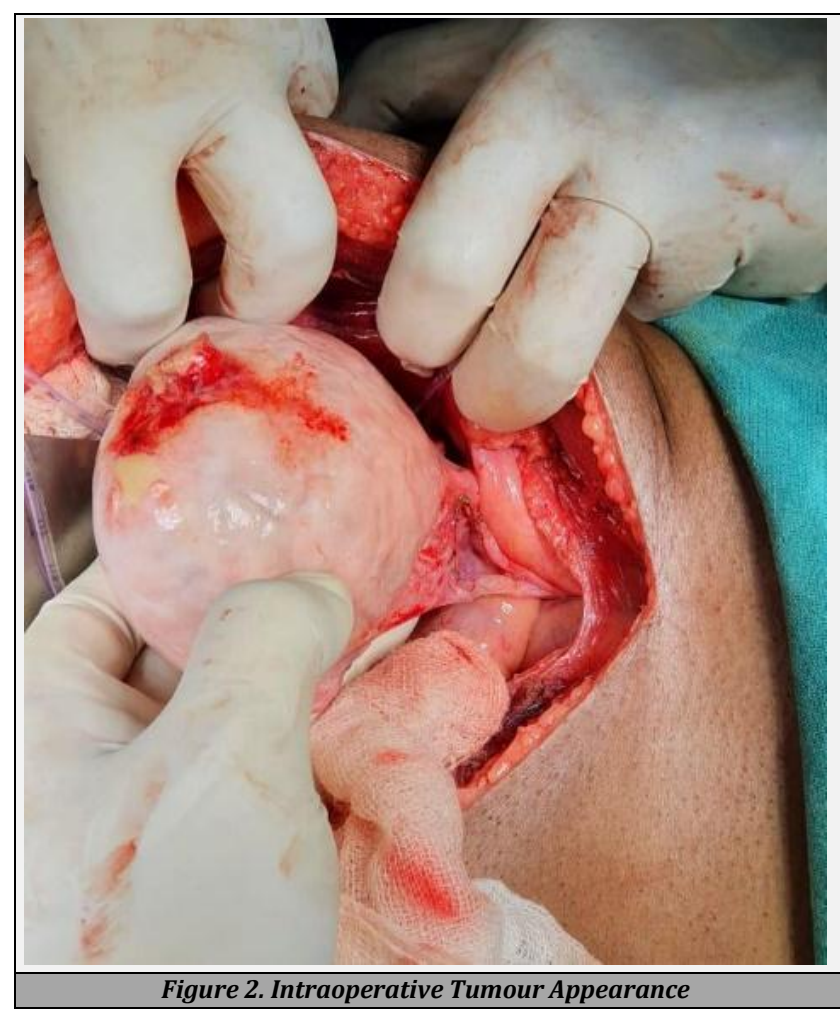

Histopathology revealed the presence of a cellular tumour with cells arranged in sheets having pleomorphic nuclei, prominent nucleoli and a moderate amount of cytoplasm (Figure 3). A diagnosis of seminoma (germ cell tumour) was made. Serum AFP and beta-HCG levels were normal.

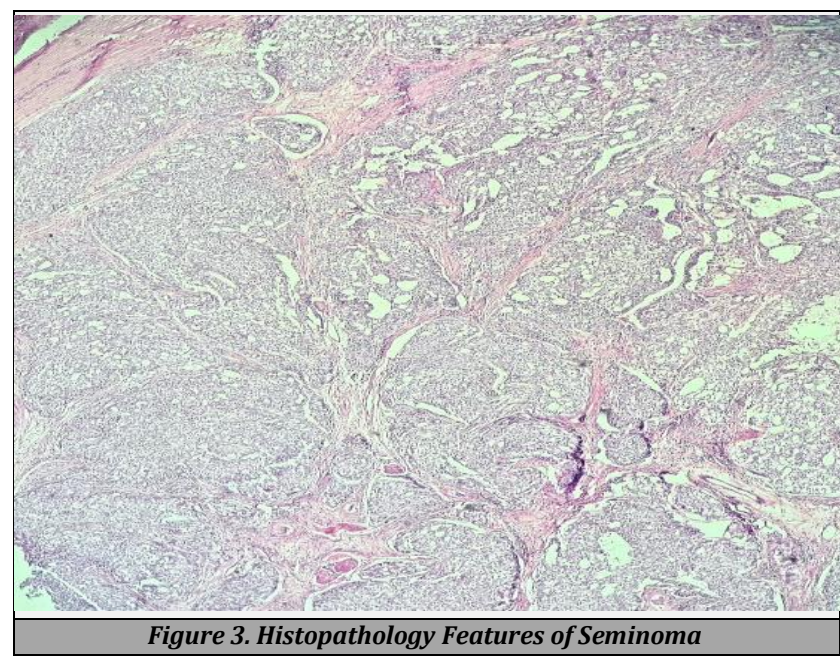




\section{DISCUSSION}

Testicular ectopia is rare and the most frequent ectopic location of testes include superficial inguinal pouch, in front and lateral to the external inguinal ring and very rarely intraabdominal. 5 The majority of undescended testes are palpable and are located distal to the external inguinal ring. ${ }^{6}$ There is a relation between the position of the undescended testis and the likelihood of carcinogenesis. The intra-abdominal testis has a higher malignant potential. Approximately seven to ten percent of testicular neoplasms develop in patients with a long-standing history of cryptorchidism. Germ cell tumours may be malignant (seminoma and non-seminoma) or benign (teratomas). ${ }^{7}$ Germ cell tumours are most common in gonads, with just two to five percent arising in extragonadal regions such as the retroperitoneum, mediastinum, sacral area and pineal gland. ${ }^{8}$ Seminoma is the commonest histologic type of germ cell tumour. They often present with lymph node metastasis. Choriocarcinoma, however, is characterized by early haematogenous dissemination. Various factors such as the age of the patient, size of the tumour, lymphovascular invasion, internal necrosis, percentage of giant cells, mitotic count and tumour infiltrating lymphocytes are possible prognostic factors that can determine the treatment of seminomas.

Determination of extension of tumour has been described in three clinical stages. Stage I tumours are limited to the testis with or without invasion of spermatic cord/epididymis. Stage II tumours are associated with retroperitoneal lymph node metastases. Stage III tumours are associated with distant metastases. In our case, the tumour was designated as stage I seminoma with the presence of lymphovascular invasion (tunica vasculosa nested tumour cells).

A unilateral, rapidly growing intra-abdominal mass lesion associated with undescended testes must alert physicians towards the possibility of seminoma, therefore, prompting early initiation of intervention. We managed a patient presenting with a progressively increasing mass in his lower abdomen with few other symptoms. Physical examination revealed the absence of one testis from his scrotum which was highly indicative of a seminoma. Given the rarity of reported cases, our case will serve to reinforce already existing literature and permit physicians in a similar setting to think of a testicular neoplasm, especially in ambiguous presentations such as ours. Imaging modalities like ultrasound, CT scan, and MRI usually show a well-defined heterogeneous mass lesion, this appearance is non-specific and can mimic other commonly observed pathologies such as significantly enlarged lymph nodes and sarcoma. ${ }^{9}$ Thus the definitive diagnosis relies upon histopathology. Surgical resection followed by a histopathologic analysis of the tumour is advocated in the majority of cases.

\section{CONCLUSIONS}

Testicular seminoma is a common neoplasm of middle-aged men with cryptorchidism being a significant risk factor. Therefore, we stress the need for a scrotal examination in addition to routine physical examination, in men presenting with an abdominal mass and with an empty scrotal sac, to rule out the possibility of an intra-abdominal tumour of undescended testes.

\section{REFERENCES}

[1] Wood HM, Elder JS. Cryptorchidism and testicular cancer: separating fact from fiction. J Urol 2009;181(2):452-61.

[2] Nkembe MN, Mvalo CM, Tianyi FL, et al. Ambiguous presentation of an intra-abdominal testicular seminoma in a 40-year-old man: a case report. J Med Case Rep 2019;13(1):2.

[3] Mayo clinic. Undescended testicle-symptoms and causes. https://www.mayoclinic.org/diseasesconditions/undescended-testicle/symptomscauses/syc-20351995.

[4] Cortes D, Thorup J, Petersen BL. Testicular neoplasia in undescended testes of cryptorchid boys-does surgical strategy have an impact on the risk of invasive testicular neoplasia? Turk J Pediatr 2004;46 Suppl:35-42.

[5] Shah M, Patel L, Bhatt C, et al. Case report: seminoma in abdominal ectopic testis. Indian J Radiol Imag 2004;14(3).

[6] Woodward PJ. Case 70: seminoma in an undescended testis. Radiology 2004;231(2):388-92.

[7] Yılmaz A, Bayraktar B, Sagiroglu J, et al. Giant seminoma in an undescended testis presenting as an abdominal wall mass. J Surg Case Rep 2011;2011(12):9.

[8] Gândara F, Bernardo M, Noronha MM, et al. P0312 mediastinal seminoma: in reference to a case report. Eur J Intern Med 2009;20:S109.

[9] Marko J, Wolfman DJ, Aubin AL, et al. Testicular seminoma and its mimics: from the radiologic pathology archives. Radiographics 2017;37(4):1085-98. 